\title{
Drawing Graphs with Nonuniform Nodes Using Potential Fields
}

\author{
Jen-Hui Chuang ${ }^{1}$, Chun-Cheng Lin ${ }^{2}$, and Hsu-Chun Yen ${ }^{2 \star}$ \\ 1 Dept. of Computer and Information Science, \\ National Chiao-Tung University, Taiwan \\ jchuang@cis.nctu.edu.tw \\ 2 Dept. of Electrical Engineering, National Taiwan University, Taiwan \\ sanlin@cobra.ee.ntu.edu.tw, yen@cc.ee.ntu.edu.tw
}

\begin{abstract}
A potential field approach, coupled with force-directed methods, is proposed in this paper for drawing graphs with nonuniform nodes in 2-D and 3-D. In our framework, nonuniform nodes are uniformly or nonuniformly charged, while edges are modelled by springs. Using certain techniques developed in the field of potential-based path planning, we are able to find analytically tractable procedures for computing the repulsive force and torque of a node in the potential field induced by the remaining nodes. Our experimental results suggest this new approach to be promising, as drawings of good quality for a variety of graphs in 2-D and $3-\mathrm{D}$ can be produced efficiently.
\end{abstract}

\section{Introduction}

As graphs are known to be one of the most important abstract models in various scientific and engineering areas, graph drawing (or information visualization in a broader sense) has naturally emerged as a fast growing research topic in computer science. In visualizing graphs associated with real-world entities, it is common to annotate each node with some labels (such as name, attribute, for instance), and a good way to display such information is to fill the information inside the drawing of the node. As a result, it is of importance and interest to be able to cope with vertices of different sizes and shapes in graph drawing.

Most of the conventional graph drawing algorithms only deal with graphs with zero-sized vertices. A naive extension of conventional drawing algorithms by plugging in objects of nonzero size for point vertices in the drawing is unlikely to produce a high quality drawing. In view of this, it becomes apparent that a new line of research has to be carried out in hope of narrowing the gap between the conventional algorithms and graphs consisting of nodes of various sizes and shapes that are frequently encountered in the real world. Along this line of research, a recent article [1] extends the conventionally force directed method [2] by developing two new algorithms (called the elliptic spring method and the modified spring method) to cope with graphs with nonuniform nodes

\footnotetext{
* Corresponding author 
(including circles, ellipses and rectangles). The underlying aesthetic criteria include preventing nonuniform nodes from being overlapped and edges from being crossed over with one another. Some encouraging results have been reported in [1].

In this paper, we present a new approach, combining the force-directed strategy as well as the theory of potential fields, for drawing graphs with nonuniform nodes. In our setting, each edge is modelled by a spring (same as that in conventional force-directed methods) and the boundary of every nonuniform node is uniformly or nonuniformly charged. We consider both 2-dimensional and 3dimensional drawings of nonuniform nodes, each of which is represented by a polygon (in 2-D) or polyhedron (in 3-D). Other shapes of objects can be approximated by their enclosing polygons (or polyhedrons). For a given nonuniform node, the forces on the node come from two sources: one resulting from being connected by springs, and the other is caused by being present in the potential field induced by the remaining nodes. In comparison with the work of [1], our method deals with graphs in not only 2-D but 3-D, and makes every nonuniform node rotate with an angle of inclination to display a high degree of symmetry.

The rest of this paper is organized as follows. In Section 2, the theory behind potential fields in graph drawing is developed in depth. Section 3 illustrates a number of experimental results. Finally, a conclusion is given is Section 4 .

\section{Force-Directed Method Using Potential Fields}

To draw graphs with nonuniform nodes, our force-directed method is based upon the idea of replacing each edge by a spring and assuming that the border of every nonuniform node is uniformly or nonuniformly charged. In this setting, the repulsive forces among nonuniform nodes avoid collision, and the spring forces pull nonuniform nodes closer. A nice drawing would be generated when the corresponding model reaches an equilibrium between the repulsive forces due to the charged nonuniform nodes and attractive forces due to springs.

In what follows, we mainly focus on the repulsive forces due to charged nonuniform nodes; the force formula of springs is based upon Hooke's Law [2] which uses the following logarithmic function as the force formula:

$$
f_{a}(d)=C 1 \log (d / C 2)
$$

where $d$ is the spring length, and $C 1$ and $C 2$ are constants. In addition to repulsive forces, the repulsive torques need also be considered since every nonuniform node has some degree of inclination.

In what follows, we elaborate on the foundations of the theory behind the use of potential fields in graph drawing. The reader is referred to 34 for more about potential fields as well as some of the detailed derivations of formulas involved in our subsequent discussion.

Consider two polygons (representing two nonuniform nodes) $A$ and $B$ in 2-D connected by two edges $\overline{a_{1} b_{2}}$ and $\overline{a_{3} b_{2}}$ as shown in Figure 1 1 (a). $A$ has vertices $a_{1}, \ldots, a_{6}$, and $B$ has $b_{1}, b_{2}$, and $b_{3}$ along their boundaries. Each polygon 


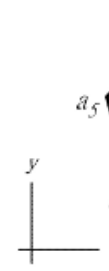

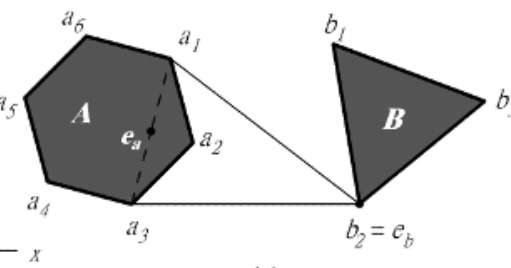

(a)

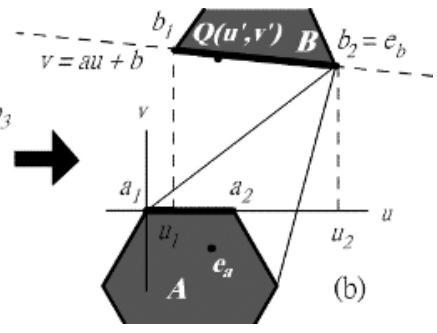

(b)

Fig. 1. Coordinate transformation of a 2-D graph with two nonuniform nodes $A$ and $B$ where $e_{a}$ and $e_{b}$ are the reference points. (a) The original coordinate system (xy-plane). (b) The new coordinate system ( $u v$-plane) after the transformation.

is associated with a reference point exerted by repulsive and attractive forces, and shifts and rotations are carried out with respect to this reference point. For instance, in Figure $1 e_{a}$ and $e_{b}$ are the reference points of $A$ and $B$, respectively. For any two points of unit charge and of distance $r$ apart, the Newtonian potential is defined as

$$
V=\frac{1}{r}
$$

and the Newtonian potential of a point $x$ with respect to a line segment $\overline{a_{1} a_{2}}$ is

$$
V=\int_{a_{1}}^{a_{2}} \frac{1}{r} d x
$$

More generally, if the charge density increases (decreases) quadratically while $x$ is moving along the line segment from $a_{1}$ to $a_{2}$, the charge density function can be expressed as $\alpha x^{2}+\beta x+\gamma$ with some constants $\alpha, \beta$, and $\gamma$; furthermore, the total potential at a point due to the charged line segment is equal to

$$
V=\int_{a_{1}}^{a_{2}} \frac{\alpha x^{2}+\beta x+\gamma}{r} d x
$$

which can be evaluated analytically (4). Note that by letting $\alpha=0$ (resp., $\alpha=\beta=0$ and $\gamma=1$ ), the above equation characterizes the linear (resp., Newtonian) potential field.

Consider two line segments $\overline{a_{1} a_{2}}$ and $\overline{b_{1} b_{2}}$ on the new coordinate system (uvplane) after coordinate transformation as shown in Figure 1 1 (b). $Q(u \prime, v \prime)$ is a point on $\overline{b_{1} b_{2}}$, and $v=a u+b$ (where $a$ and $b$ are constants) is the line equation representing $\overline{b_{1} b_{2}}$. $d$ is the length of $\overline{a_{1} a_{2}}$, and $u_{1}$ and $u_{2}$ are the projection points of the end points of $\overline{b_{1} b_{2}}$. After a sequence of computations of the potential function of Eq. 4. such as negative gradient, integral, and coordinate transformation, the repulsive force on $\overline{b_{1} b_{2}}$ due to $\overline{a_{1} a_{2}}$, i.e. the repulsive force between two line segments, can be formulated as 1

\footnotetext{
${ }^{1}$ For simplicity, only the u-component is considered for the rest of this paper.
} 


$$
F_{u}=\sqrt{1+a^{2}} \int_{u_{2}}^{u_{1}} \int_{0}^{d} \frac{u^{\prime}-u}{r^{3}} \rho(u) \rho\left(u^{\prime}\right) d u d u^{\prime} .
$$

And the repulsive torque on $\overline{b_{1} b_{2}}$ due to $\overline{a_{1} a_{2}}$, i.e. the repulsive torque between two line segments, with respect to the reference point $e_{b}$ can be formulated as

$$
\begin{aligned}
\tau_{e_{b}}= & \sqrt{1+a^{2}}\left(\int_{u_{2}}^{u_{1}} \int_{0}^{d}\left(u^{\prime}-u_{0}\right) \frac{a u^{\prime}+b}{r^{3}} \rho(u) \rho\left(u^{\prime}\right) d u d u^{\prime}\right. \\
& \left.-\int_{u_{2}}^{u_{1}} \int_{0}^{d}\left(a u^{\prime}+b-v_{0}\right) \frac{u^{\prime}-u}{r^{3}} \rho(u) \rho\left(u^{\prime}\right) d u d u^{\prime}\right)
\end{aligned}
$$

Assume that the charge density $\rho(u)$ is equal to $1, u$, or $u^{2}$ for a border line $\overline{a_{1} a_{2}}$ of $A$, and $\rho(s)=1, s$, or $s^{2}$ for a border line $\overline{b_{1} b_{2}}$ of $B$, nine different combinations of charge distributions need to be considered in evaluating the repulsive force and torque between the two line segments. For example, form (5), the repulsive force along the $u$-axis for these nine combinations can be obtained from

$$
\begin{aligned}
F_{u}^{i j} & =F_{u}^{i j}\left(u_{2}\right)-F_{u}^{i j}\left(u_{1}\right) \\
& =\left(1+a^{2}\right)^{\frac{j+1}{2}} \int_{u_{1}}^{u_{2}} \int_{0}^{d} \frac{u^{\prime}-u}{r^{3}} u^{i} d u\left(u^{\prime}\right)^{j} d u^{\prime}
\end{aligned}
$$

where $i(=0,1$, or 2$)$ is equal to the order of the charge density of the border line $\overline{a_{1} a_{2}}$, and $j(=0,1$, or 2$)$ is equal to that of the border line $\overline{b_{1} b_{2}}$. It can be shown that analytic expressions exist for all these integral equations. Similar results can be obtained for $F_{v}$ and $\tau_{e_{b}}$.

In addition, the torques due to the spring forces should be considered. In Figure 11. suppose the attractive force on the point $b_{2}$ due to the spring edge $\overline{a_{1} b_{2}}$ is equal to $f_{a}(d)$, where $d$ is the length of $\overline{a_{1} b_{2}}$ from (1), and $B$ rotates with respect to the reference point $e_{b}$. The torque with respect to point $e_{b}$ due to the attractive force $f_{a}(d)$ from spring $\overline{a_{1} b_{2}}$, on point $b_{2}$, is equal to

$$
T_{e_{b}}=f_{a}(d) \times\left(b_{2}-e_{b}\right)
$$

which is perpendicular to the plane containing $A$ and $B$ (i.e., the $x-y$ plane).

Note that it is inappropriate to choose the center of shape as the reference point of a polygon because the forces and torques due to springs are computed by the spring lengths and each spring is not connected to the center of shape. Instead, the mean of the coordinates of the vertices connected by springs is selected as the reference point. Take Figure 1 for instance. $A$ has vertices $a_{1}$ and $a_{3}$ connected by springs, and the midpoint of $a_{1}$ and $a_{3}$ is the reference point of $A$. $B$ only has a single vertex $b_{2}$ connected by a spring, and hence $b_{2}$ is the reference point of $B$. 


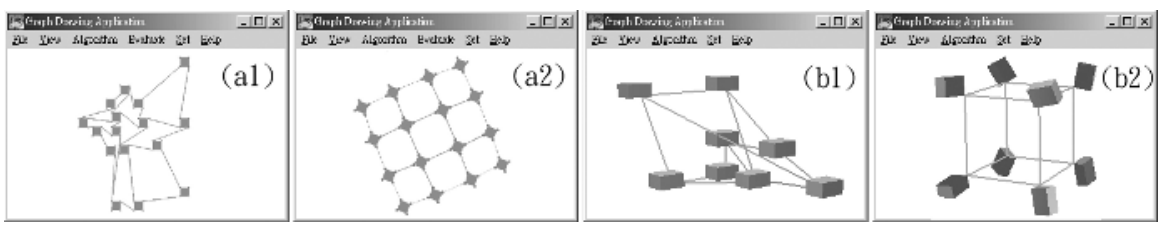

Fig. 2. (a) Mesh structure in 2D. (b) Cube structure in 3D. (a1, b1: initial drawings; $a 2, b 2$ : final drawings)

In view of the above derivations, the force on a line segment due to a polygon can be derived. For example, in Figure 1, the total repulsive force on polygon $B$ due to polygon $A$ is equal to

$$
F_{u}^{r}=\sum_{n \in \mathbf{B}(B)} F_{u}^{n}=\sum_{n \in \mathbf{B}(B)} \sum_{m \in \mathbf{B}(A)} F_{u}^{n m}
$$

where $n$ is a border line of $B, m$ is a border line of $A, \mathbf{B}(A)$ is the set of border lines of $A$, and $F_{u}^{m n}$ is the repulsive force on line segment $n\left(\overline{b_{1} b_{2}}\right)$ due to $m$ along the $u$-axis from (7). On the other hand, the total attractive force on polygon $B$ due to all of the springs is equal to

$$
F_{u}^{a}=\sum_{p \in \mathbf{P}(B)} F_{u}^{p}=\sum_{p \in \mathbf{P}(B)} \sum_{e \in \mathbf{E}(G)} f_{a u}(d(e))
$$

where $p$ is a vertex of $B, e$ is an edge of the graph, $\mathbf{E}(G)$ is the set of edges of the graph $G, d(e)$ is the length of spring edge $e, \mathbf{P}(B)$ is the set of vertices of $B$, and $f_{a u}$ is the spring force defined in (11) along the $u$-axis. In summary, the total force applied to polygon $A$ due to the remaining polygons and springs is equal to the sum of the repulsive force (Eq. 9) and the attractive force (Eq. 10).

$$
\mathbf{F}_{u}=\sum_{\mathbf{V}-\{B\}}\left(F_{u}^{r}+F_{u}^{a}\right)
$$

Similar results can be obtained for the total force $\mathbf{F}_{v}$ along the $v$-axis and the total torque $\tau_{e_{b}}$ with respect to the reference point $e_{b}$.

Due to space limitations, the details of extending the potential field approach to $3 \mathrm{D}$ are omitted here.

\section{Experimental Results}

Based on the theory of potential fields detailed in the previous section, we develop a prototype system for drawing graphs with nonuniform nodes in 2D and 3D.

Figure 2 shows some drawing results in 2D and 3D. Figure 3 illustrates a strategy to handle the drawing of a clustered graph [5]. (See [6] for a related result.) 


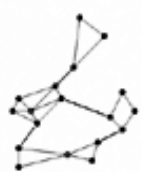

(a)

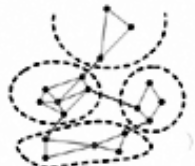

(b)

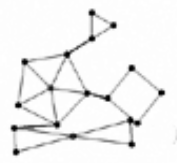

(c)

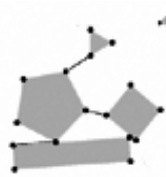

(d)

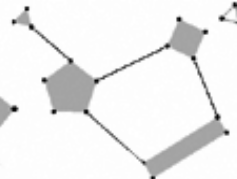

(e)

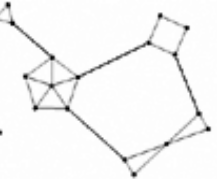

(f)

Fig. 3. Drawing a clustered graph. (a) A clustered graph. (b) Cluster partition. (c) Drawing individual clusters using the conventional force-directed method. (d) Convex hulls of clusters. (e) Drawing while treating clusters as nonuniform nodes. (f) Final drawing by plugging in details of clusters.

\section{Conclusion}

A potential-based approach, coupled with a force-directed method, has been proposed and implemented for drawing graphs with nodes of different sizes and shapes. A unique feature of our approach is that in the process of reaching equilibrium, the degree of inclination of a nonuniform can be adjusted while moving from one position to another. By doing so, the final drawing has a tendency to display a high degree of symmetry. An equally important aspect of our approach is that the formulas derived in our potential field model are analytically tractable, making our algorithm computationally efficient. Our experimental results look promising. It would also be of interest to compare our approach with existing algorithms designed specially for drawing graphs with nonuniform nodes, in spite of the scarcity of such algorithms.

\section{References}

1. D. Harel and Y. Koren. Drawing graphs with non-uniform vertices. Proc. of Working Conference on Advanced Visual Interfaces (AVI'02), pp. 157-166, ACM Press, 2002.

2. P. Eades. A heuristic for graph drawing. Congress Numerantium, 42, pp. 149-160, 1984.

3. J.-H Chuang and N. Ahuja. An analytically tractable potential field model of free space and its application in obstacle avoidance. IEEE Transactions on System, Man, and Cybernetics - Part B: Cybernetics, Vol. 28, No. 5, pp. 729 -736, Oct, 1998.

4. J.-H Chuang. Potential-based modeling of three-dimensional workspace for obstacle avoidance. IEEE Trans. Robotics and Automation, Vol. 14, No. 5, pp. 778-785, Oct. 1998.

5. P. Eades and M. L. Huang. Navigating clustered graphs using force-directed methods. Journal of Graph Algorithms and Applications, Vol. 4, No. 3, pp. 157-181, 2000 .

6. M. Huang, P. Eades and J. Wang. On-line animated visualization of huge graphs using a modified spring algorithm. Journal of Visual Languages and Computing, pp. 623-345, 1998. 